\title{
SIGNAL TRANSDUCTION IN FUNGI - THE ROLE OF PROTEIN PHOSPHORYLATION
}

\author{
Z. FEHÉR, and KRISZTINA SZIRÁK
}

Department of Human Genetics, University Medical School of Debrecen, Debrecen, Hungary

Living cells are able to respond to the surrounding environment. As a first step in this process, membrane receptors react with an extracellular ligand. There are three main families of cell-surface receptors: (1) Ion-channel-linked receptors, (2) G-protein-linked receptors, and (3) Enzyme-linked receptors that either act directly as enzymes or are associated with enzymes. These enzymes are often protein kinases that phosphorylate specific proteins in the target cell. Through cascades of phosphorylations elaborate sets of proteins relay signals from the receptor to the nucleus regulating gene expression. There are two groups of protein kinases: tyrosine- and serine-threonine-specific protein kinases and there are protein phosphatases with specificity for the appropriate side chain to match each type of kinase. They can terminate an activation event reversing the phosphorylation caused by a protein kinase.

Receptors, effectors, second messengers, kinases, phosphatases and transcriptional regulators form signal transduction pathways, relaying a specific signal to the gene expression machinery or other target sites thus change the cell's behavior. One can recognize significant similarities in signal transduction pathways of lower and higher eukaryotes. E.g. all eukaryotes transduce external signals via a conserved module composed of three protein kinases, the mitogen activated protein kinase (MAPK) cascade. Another "classical" pathway includes G-proteins, adenylate cyclase, cAMP and protein kinase A. In this lecture we summarize some of the important fungal signal transduction pathways and briefly describe our own work in the field of Neurospora crassa protein phosphatases.

In eukaryotic cells the MAPK cascade lies in the heart of many signalling pathways. Three protein kinases that are highly conserved in all eukaryotes make up this module: MAPK (also known as extracellular signal-regulated kinase [ERK]), MAPK kinase, (MAPKK, also known as mitogen-activated, ERK-activating kinase [MEK]) and MAPK kinase kinase (MAPKKK, also known as MEK kinase [MEKK]). The budding yeast Saccharomyces cerevisiae has at least six identified MAPK pathways that regulate response to high or low osmolarity, pheromones, perturbation of cell wall integrity, sporulation and pseudohyphal growth. In Schizosaccharomyces pombe the pathways responding to pheromones and osmotic stress were characterized $[1,2]$.

ZSIGMOND FEHÉR, KRISZTINA SZIRÁK

Department of Human Genetics, University Medical School of Debrecen

P.O. Box 1, H-4012 Debrecen, Hungary 
Adaptation to high osmolarity is mediated by the HOG (high-osmolarity glycerol) pathway in $S$. cerevisiae and the Styl (suppressor of tyrosine phosphatase) pathway in $S$. pombe. The PKC (protein kinase C) pathway is activated by low osmolarity in budding yeast. In both cases the MAPK cascade is activated, which regulates the expression of specific target genes via transcription factors. The direct target of the PKC MAPK cascade is a transcriptional activator $(\mathrm{R} \operatorname{lm} 1)$ that may regulate the expression of genes for cell wall biosynthesis.

Salt tolerance and maintenance of cell integrity are regulated by associated pathways in yeasts. Two genes, PPZ1 and PPZ2, encoding "novel type" Ser/Thr phosphatases were described, whose overexpression suppressed $\triangle m p k 1$ (MAPK) and $\Delta p k c 1$ mutations. Deletion of both genes resulted in a temperature dependent cell lysis defect, similar to that observed for MAPK cascade mutants. It was assumed that PKC pathway and $P P Z 1 / 2$ may have a common target responsible for the maintenance of cell integrity. $P P Z 1$ is also an important determinant of salt tolerance and caffeine sensitivity [3]. Functional homologues of Ppzl with striking similarities were also cloned from $S$. pombe and $N$. crassa. All three proteins have a protein phosphatase 1-like C-terminal catalytic domain and an N-terminal domain, in which the first fifty amino acid residues exhibit certain degree of similarity.

The pheromone response pathway of $S$. cerevisiae mediates cell-cell interaction during mating. This is one of the best-understood signalling pathways in eukaryotes. Studies with this yeast pathway provided a framework for understanding the effect of growth factors on cell cycle progression in mammalian cells. The most important characteristics of this pathway are the following: (1) Peptide pheromones interact with serpentine receptors and initiate the response. (2) Receptors interact with heterotrimeric G protein that transmits the signal to downstream effectors. (3) MAPKs mediate the activation of a transcription factor (Ste12). (4) One of the MAPKs phosphorylates a cyclin dependent kinase inhibitor (CKI), leading to cell cycle arrest. (5) A component of the pathway (Ste5) acts as a scaffold that brings together cascade components and prevents cross talk. (6) Many of the target genes are necessary for cell and nuclear fusion and for cell cycle arrest $[1,2]$.

There are relatively few data on signal transduction systems operating in filamentous fungi. Germination of conidia is an important developmental event in fungal life. Extra- and intracellular cues trigger the major transitions that occur during this process. A protein likely to mediate the response to the signals activating conidial germination is the cAMP-dependent protein kinase A (PKA) [4].

Most steps in signal transduction pathways involve protein phosphorylation as a signal relaying mechanism. A signal, which was generated by extra- or intracellular changes has to be terminated after some time. Protein phosphatases are responsible for this termination event: dephosphorylate the target proteins reversing the action of protein kinases. Eukaryotic protein phosphatases are classified as Ser/Thr, Tyr and dual specificity phosphatases. Ser/Thr phosphatases are divided into four subclasses (PP1, PP2A, PP2B, PP2C) on the basis of their substrate specificity, metal ion dependence and inhibitor sensitivity. On the basis of their sequence-homology, PP1, PP2A and PP2B belong to the PPP family, whereas PP2C and the related mitochondrial pyruvate dehydrogenase phosphatase are members of the PPM family [5]. Among fungi, protein 
Ser/Thr phosphatases are well known in the yeasts, considerable information is available in $N$. crassa and A. nidulans, but little is known about other fungal species. Most of the "classical" type fungal protein phosphatases play an indispensable role in the regulation of cell cycle, growth, and morphogenesis.

More recently, many other phosphatases have been discovered that do not fit into the classical categories, although they show considerable homology to some of them. These are the "novel type" protein phosphatases. They are grouped according to the sequence similarity they show to PP1 and PP2A. PP5 seems to be a third, not very closely related subfamily [6]. Three genes encoding classical type (PP1, PP2A, PP2B) and two encoding novel type phosphatases (PPT/PP5 and PPZ-like) were cloned from N. crassa to date.

\section{REFERENCES}

1. Banuett,F.: Signalling in the yeasts: An informational cascade with links to the filamentous fungi. Microbiol Mol Biol Rev 62, 249 (1998).

2. Herskowitz,I:: MAP kinase pathways in yeast: For mating and more. Cell 80, 187 (1995).

3. Posas,F., Camps,M., Arino,J.: The PPZ protein phosphatases are important determinants of salt tolerance in yeast cells. J Biol Chem 270, 13036 (1995).

4. d'Enfert,C.: Fungal spore germination: Insights from the molecular genetics of Aspergillus nidulans and Neurospora crassa. Fung Genet Biol 21, 163 (1997).

5. Barford,D.: Molecular mechanisms of the protein serine/threonine phosphatases. Trends Biochem Sci 21 , 407 (1996).

6. Cohen,P.T.W.: Novel protein serine/rhreonine phosphatases: variety is the spice of life. Trends Biochem Sci 22, 245 (1997). 